\title{
Cardioplegia Application with A Hand-Squeezed Cardioplegia Bag. Is It Safe?
}

\author{
Karaarslan K, ${ }^{1}$ Abud B, ${ }^{1}$ Karacelik M, ${ }^{2}$ Bilen C ${ }^{2}$ \\ ${ }^{1}$ University of Health Sciences Izmir Tepecik Research and Education Hospital, Cardiovascular Surgery Department, Izmir, Turkey; \\ ${ }^{2}$ University of Health Sciences Izmir Dr. Behcet Uz Children's Hospital, Department of Pediatric Cardiac Surgery, Izmir, Turkey
}

\section{ABSTRACT}

Objectives: Cardioplegia solutions have a role not only in arresting the heart but also in protecting the myocardium from ischemia. While antegrade cardioplegia is given by the heart-lung machine in many centers, it is given by a handsqueezed bag in very few centers. The pressure of cardioplegia given antegrade from the heart-lung machine is certain (60-90 $\mathrm{mmHg})$. The pressure applied in the cardioplegia method, which is given antegrade with a hand-squeezed bag, is uncertain and variable. We compared the antegrade cardioplegia method applied with a hand-squeezed bag with the antegrade cardioplegia method applied with a roller pump from the heart-lung machine in terms of protecting the myocardium from ischemia.

Methods: Seventy-six patients who did not have an acute myocardial infarction, had normal preoperative cardiac marker (troponin and CK-MB) values, did not undergo redo open heart surgery, had an ejection fraction of $50 \%$ and above, and underwent elective two or three-vessel isolated coronary artery bypass surgery were evaluated. While tepid $\left(30-32^{\circ} \mathrm{C}\right)$ blood cardioplegia was administered antegrade to 33 patients (Group A) with a hand-squeezed bag, the other 34 patients (Group B) received tepid $\left(30-32^{\circ} \mathrm{C}\right)$ antegrade blood cardioplegia from the heart-lung machine. The perioperative and postoperative data of the patients were recorded and compared. To evaluate myocardial damage, postoperative cardiac markers and echocardiography data were evaluated and compared at the fourth hour after the cross-clamp was removed in both groups.

Results: When evaluated in terms of preoperative demographic data, preoperative mean $\mathrm{EF}$ values and intraoperative data, there was no statistical difference between both groups. When we evaluated in terms of myocardial protection, the mean TnT level was $4.31 \pm 1.95$ at the 4th hour in Group A and 3.91 \pm 1.69 in Group B. Mean 4th hour CK-MB level was $40.84 \pm 9.07$ in Group A and $38.56 \pm 8.07$ in Group B. Mean change in $\mathrm{EF}(\%)$ was $-4.09 \pm 4.41$ in Group A and $3.53 \pm 4.53$ in Group B. In line with the current data when we evaluated in terms of myocardial protection, we found that there is no

Received April 28, 2021; accepted May 25, 2021.

Correspondence: Burcin Abud, Gaziler cd. No 468, Izmir Tepecik Research and Education Hospital, Department of Cardiovascular Surgery, 35280 Yenişehir, Izmir, Turkey, Telephone +90-232-4696969, GSM +90-5052168801, Fax +90-232-4330756 (e-mail: burcinabud@hotmail.com). statistical difference between the two groups $(P=0.373 ; P=$ $0.158 ; P=0.523)$. There was no statistical difference between both groups, in terms of postoperative arrhythmias. None of the patients died, and no patients required an intra-aortic balloon pump.

Results: As a result of our study, cardioplegia administration with a certain constant pressure from the roller pump and hand-squeezed bag with uncertain pressure does not make a difference, in terms of myocardial protection. We think that the content and amount of cardioplegia and the preferred time for repeated cardioplegia applications are more important for the protection of the myocardium.

\section{INTRODUCTION}

Cardioplegia application is important in cardiac surgeries performed with cardiac arrest. Cardioplegia solutions have a role not only in arresting the heart but also in protecting the myocardium from ischemia [Westaby 1997]. Cardioplegias can vary both in type (blood or crystalloid) and content [Buckberg 2016]. Also, they can be applied as antegrade or retrograde cardioplegia at different temperatures [Candilio 2014]. Cardioplegia is applied at different pressure values in both applications. In antegrade application, cardioplegia is given with a pressure of $60-90 \mathrm{mmHg}$ and a rate of $200-$ $350 \mathrm{ml} / \mathrm{min}$; while in the retrograde application, cardioplegia is given with a pressure of $40 \mathrm{mmHg}$ and a rate of $250-300$ $\mathrm{ml} / \mathrm{min}$ [Kirali 2006]. While antegrade cardioplegia is given by the heart-lung machine in many centers, it is given by a hand-squeezed bag in very few centers [Kirali 2006]. While the pressure of cardioplegia given antegrade from the heartlung machine is certain $(60-90 \mathrm{mmHg})$, the pressure applied in the cardioplegia method, which is given antegrade with a hand-squeezed bag, is uncertain and variable.

In this study, we compared the antegrade cardioplegia method applied with a hand-squeezed bag with the antegrade cardioplegia method applied with a roller pump from the heart-lung machine in terms of protecting the myocardium from ischemia.

\section{MATERIAL AND METHOD}

In our clinic, between 2010 and 2012, cardioplegia was administered antegrade with a hand-squeezed bag. After 2012, cardioplegia is administered antegrade from the heartlung machine with a roller pump. Two hundred forty-six 
Table 1. Preoperative and postoperative exclusion criteria
1. Patient age lower than 30 and higher than 80
2. Existence of preoperative medically treated renal disease or COPD
3. Existence of preoperative hematological dysfunction
4. Preoperative warfarin and/or fibrinolytic agent treatment
5. Acute MI with emergent surgery
6. Re-operations for CABG
7. Concomitant valve and/or vascular surgery
8. Existence of preoperative atrial fibrillation
9. Off-pump CABG operations
10. LVEF worse than $50 \%$
11. Postoperative severe neurologic and/or other systemic complications
12. Reoperations for early massive bleeding after surgery
13. Patients with preoperative high troponin levels

CABG, coronary artery bypass graft; COPD, chronic obstructive pulmonary disease; LVEF, left ventricle ejection fraction; MI, myocardial infarction

Table 2. Inclusion criteria

1. Patients only on pump CABG with 2 or 3 vessel bypasses

2. Patients with normally cardiac segment functions ( $E F \geq 50 \%$ )

3. Patients with normally preoperative TnT and CK-MB levels (without preoperative MI)

CABG, coronary artery bypass graft; EF, ejection fraction; $\mathrm{TnT}$, Troponin T; CK-MB, Creatine kinase myocardial isoenzyme; MI, myocardial infarction

Table 3. Demographic data of the patients

\begin{tabular}{lccc}
\hline & Group A (N=33) & Group B (N=34) & P-value \\
\hline Mean age & $63.33 \pm 11.09$ & $60.44 \pm 10.68$ & 0.248 \\
Male/female & $19 / 14$ & $18 / 16$ & 0.703 \\
Diabetes mellitus (n) & $9(27 \%)$ & $12(35 \%)$ & 0.479 \\
HPL (n) & $7(21 \%)$ & $6(18 \%)$ & 0.712 \\
Preoperative EF (\%) & $54.70 \pm 4.5$ & $55.88 \pm 4.68$ & 0.282 \\
\hline
\end{tabular}

$\mathrm{HPL}$, hyperlipidemia; EF, ejection fraction

patients, who underwent coronary artery bypass surgery in our clinic between 2010 and 2014, were evaluated. Patients between the ages of 30 and over 80 , and patients with kidney disease, chronic obstructive pulmonary disease, hematological dysfunction, and preoperative atrial fibrillation were not included in the evaluation. Patients who used warfarin/ fibrinolytic agents before surgery and those with additional cardiac valve dysfunction were excluded from the evaluation. (Table 1) Patients who did not have an acute myocardial infarction, had normal preoperative cardiac marker (troponin and CK-MB) values, did not undergo redo open heart surgery, had an ejection fraction (EF) of $50 \%$ and above, and underwent elective two or three-vessel isolated coronary artery bypass surgery were evaluated. (Table 2) Among these patients, patients who developed intraoperative complications, and those who developed neurological dysfunction or other systemic complications in the postoperative period were excluded from the study.

Patients who were reoperated because of bleeding in the postoperative period were also excluded (Table 1). As a result, 76 patients who were operated by the same two experienced surgeons were evaluated. While tepid $\left(30-32^{\circ} \mathrm{C}\right)$ blood cardioplegia was administered antegrade to 33 patients (Group A) with a hand-squeezed bag, the other 34 patients (Group B) received tepid $\left(30-32^{\circ} \mathrm{C}\right)$ antegrade blood cardioplegia from the heart-lung machine.

While antegrade cardioplegia was given with a handsqueezed bag, pressure control was evaluated only by palpation of the ascending aorta in the surgical field, while antegrade cardioplegia was given from the heart-lung machine with a constant pressure $(60-90 \mathrm{mmHg})$ and flow $(200-350 \mathrm{ml} /$ min). Ascending aortic palpation in the surgical field was performed only for control purposes. The perioperative and postoperative data of the patients were recorded and compared. To evaluate myocardial damage, postoperative cardiac markers and echocardiography data were evaluated and compared at the fourth hour after the cross-clamp was removed in both groups.

Coronary artery bypass surgery and cardioplegia application: Conventional coronary artery bypass surgery and cardioplegia were applied to all patients. After graft preparation and standard cannulation, cardiopulmonary bypass and cooling were started. A cross-clamp was placed at $32^{\circ} \mathrm{C}$. After placing the cross-clamp, both groups received antegrade tepid blood cardioplegia in approximately 3 to 4 minutes. Antegrade tepid blood cardioplegia was given at a dose of $20 \mathrm{ml} / \mathrm{kg}$, that resulted with cardiac arrest.

While antegrade cardioplegia was given to the patients in Group A with a manual squeezing bag, antegrade cardioplegia was given to the patients in Group B with a roller pump from the heart-lung machine. LIMA-LAD bypass was applied to all patients. The saphenous vein was used as a graft in the other coronary artery bypasses. Repeated doses of the cardioplegia were administered at a dose of $10 \mathrm{ml} / \mathrm{kg}$ every 20 minutes. After coronary artery distal bypass procedures, the cross-clamp was removed, by warming up at $34^{\circ} \mathrm{C}$. Proximal anastomoses of the vein grafted bypasses were made with the side clamp to the ascending aorta.

Cardioplegia content:

Tepid $\left(30-32^{\circ} \mathrm{C}\right)$ blood cardioplegia with the same content was applied to all patients.

- $600 \mathrm{ml} 0.9 \% \mathrm{NaCL}$

- $60 \mathrm{mEq} 7.5 \% \mathrm{KCL}$

- $20 \mathrm{mEq} \mathrm{NaHCO} 38.4$ molar\%

- $24 \mathrm{mEq} \mathrm{MgSO} 4$ 15\%

- 4: $1 \mathrm{blood} /$ cardioplegia dilution provided

The number of electrolytes in repeated cardioplegia was 
Table 4. Patients' perioperative data

\begin{tabular}{lccc}
\hline & Group A $(N=33)$ & Group B $(N=34)$ & $P$-value \\
\hline Cross-clamp time (mean) & $40.58 \pm 8.92$ & $38.82 \pm 8.60$ & 0.379 \\
CPB time (mean) & $69.67 \pm 11.93$ & $65.71 \pm 9.47$ & 0.091 \\
Number of grafts per patient (mean) & 2.61 & 2.59 & 0.883 \\
Positive Inotrope requirement at the end of CPB $(n)$ & $8(24 \%)$ & $0(0 \%)$ & $0(0 \%)$ \\
Interoperative mortality (n) & $0(0 \%)$ & $0(0 \%)$ \\
IABP requirement at the end of CPB $(n)$ & & - \\
\hline
\end{tabular}

CPB, cardiopulmonary bypass; IABP, intra-aortic balloon pump

Table 5. Postoperative data of the patients

\begin{tabular}{lccc}
\hline & Group A (N=33) & Group B (N=34) & $P$-value \\
\hline 4th hour TnT level (mean) & $4.31 \pm 1.95$ & $3.91 \pm 1.69$ & 0.373 \\
4th hour CK-MB level (mean) & $40.84 \pm 9.07$ & $38.56 \pm 8.07$ & 0.158 \\
EF change (\%) (mean) & $-4.09 \pm 4.41$ & $-3.53 \pm 4.53$ & 0.523 \\
Postoperative mortality (n) & $0(0 \%)$ & $0(0 \%)$ & - \\
Postoperative IABP requirement (n) & $0(0 \%)$ & $7(0 \%)$ & - \\
Supraventricular arrhythmia (n) & $9(27 \%)$ & $7(21 \%)$ & 0.521 \\
Ventricular arrhythmia (n) & $3(9 \%)$ & $5(12 \%)$ & 0.709 \\
\hline
\end{tabular}

TnT, Troponin T; CK-MB, Creatine kinase myocardial isoenzyme; EF, ejection fraction; IABP, intra-aortic balloon pump

reduced by half the dose.

Statistical analysis: All the data obtained from the study were analyzed with Statistical Package for the Social Sciences (SPSS Inc., Chicago, Illinois, USA) 16.0 version for Windows. Chi square test or the Fisher's exact test was used for the nominal data, while numerical data were compared using Mann-Whitney U test. A $P$ value less than $0.05(P<0.05)$ was considered statistically significant.

\section{RESULTS}

As a result of our study, 76 patients were included in the study. Nineteen men and 14 women in Group A and 18 men and 16 women in Group B were included in the study. The mean age of the patients in Group A was $63.33 \pm 11.09$, and the mean age of the patients in Group B was $60.44 \pm 10.68$. When evaluated in terms of preoperative demographic data and preoperative mean EF values, there was no statistical difference between the groups. (Table 3) When intraoperative data were evaluated, it was seen that there was no statistical difference between the two groups, in terms of cross-clamp times and cardiopulmonary bypass times. Furthermore, there was no statistically significant difference between both groups, in terms of the number of bypassed grafts and the need for inotropes when cardiopulmonary bypass was terminated. In Group A, the mean number of bypassed grafts was 2.61, and in Group B, the mean number of bypassed grafts was 2.59 . When cardiopulmonary bypass was terminated in Group A, $24 \%$ of the patients needed positive inotrope, while $32 \%$ of the patients in Group B needed positive inotrope. (Table 4) None of the patients died and no one required an intra-aortic balloon pump. When we evaluated in terms of myocardial protection, the mean $T n T$ level was $4.31 \pm 1.95$ at the fourth hour in Group A, while fourth-hour mean TnT level was $3.91 \pm 1.69$ in Group B. Mean fourth-hour CK-MB level was $40.84 \pm 9.07$ in Group A and $38.56 \pm 8.07$ in Group B. Mean change in $\mathrm{EF}(\%)$ was $-4.09 \pm 4.41$ in Group A and $3.53 \pm 4.53$ in Group B. In line with the current data when we evaluated in terms of myocardial protection, we found that there is no statistical difference between the two groups $(P=0.373 ; P=$ $0.158 ; P=0.523)$. There was no statistical difference between both groups, in terms of postoperative arrhythmias. (Table 5)

\section{DISCUSSION}

The first attempt at cardioplegia was described in 1955 by Melrose, who observed that blood with high concentrations of potassium citrate could be used for "elective 
reversible cardiac arrest." Cardioplegia has enabled the heart to stop in diastole with its high potassium content, and thus open-heart surgeries can be performed in the standing heart [Melrose 1955]. Potassium provides the advantage that open-heart surgery can be performed in the arrested heart; with the cardiac arrest, it provided an approximately $90 \%$ reduction in myocardial oxygen consumption. Thus, cellular utilization of high-energy phosphates also has been reduced and the risk of myocardial ischemia has tried to be prevented [Chambers 2003]. The causes of myocardial damage that may occur in open-heart surgery are myocardial ischemia and reperfusion injury [Buckberg 1987]. Reperfusion injuries can lead to a variety of outcomes, from "myocardial stunning" to fatal damage. Although the first type of cardioplegia described was blood cardioplegia, the commonly used type of cardioplegia was crystalloid cardioplegia in the first years. Due to Buckberg's positive views on blood cardioplegia in 1987, blood cardioplegia was started to be preferred [Buckberg 1987]. Currently, both types of cardioplegia are preferred. We prefer blood cardioplegia in our clinic. The hemoglobin contained in blood cardioplegia has oxygen-carrying properties. Therefore, high energy phosphate is produced by aerobic metabolism. Furthermore, it has the buffering ability with blood proteins, and the hemodilution seen in crystalloid cardioplegia does not occur. Besides, reperfusion damage is less common with free oxygen radical scavengers present in large amounts in erythrocytes [Beyesdorf 1999]. Cardioplegia applications also differ, according to the centers in terms of heat. While some centers prefer warm blood cardioplegia $\left(34-37^{\circ} \mathrm{C}\right)$, some centers prefer tepid $\left(30-32^{\circ} \mathrm{C}\right)$ or cold $\left(4-10^{\circ} \mathrm{C}\right)$ blood cardioplegia. In a meta-analysis published by Abah et al. in 2012 and a meta-analysis conducted by Fan et al. in 2010, it was found that the preferred temperature when using blood cardioplegia, does not have a significant difference in terms of the development of mortality, myocardial infarction, low cardiac output syndrome, atrial fibrillation, and intra-aortic balloon pump requirement [Abah 2012; Fan 2010]. In our clinic, between 2010 and 2014, tepid blood cardioplegia $\left(30-32^{\circ} \mathrm{C}\right)$ was preferred. After Calafiore et al. mentioned delivering blood cardioplegia from the heartlung machine using a roller pump, this method was used worldwide [Calafiore 1995]. Apart from that, some centers apply cardioplegia with a hand-squeezed bag. In these centers, cardioplegia is created by preparing the blood taken from the arterial line in a bag. Antegrade cardioplegia is usually applied by the heart-lung machine in many centers, while in some centers it is applied with a hand-squeezed bag [Kirali 2006; Yilik 2004]. While the pressure of the cardioplegia given from the heart-lung machine is certain $(60-90 \mathrm{mmHg})$, the pressure of the cardioplegia, which is given with a hand-squeezed bag, is uncertain and variable.

Several studies on the optimal infusion pressure of cardioplegia have shown that higher infusion pressure in cardioplegia leads to faster cooling, faster cardiac arrest, and increased cardioplegic flow through the distal coronary stenosis [Grover 1982]. Between 2010 and 2012, antegrade cardioplegia was applied with a hand-squeeze bag in our center.
After 2012, we started to apply the antegrade cardioplegia with a certain pressure from the heart-lung machine via a roller pump. There is only one study in the literature comparing the antegrade application of cardioplegia with a handsqueezed bag and the antegrade application of cardioplegia with the heart-lung machine, in terms of protection of the myocardium. This study is the animal experiment conducted by Grover et al. Grover et al. divided 14 dogs into two groups and applied cardioplegia at $30^{\circ} \mathrm{C}$ to one group with a handsqueezed bag, and the other group at $30^{\circ} \mathrm{C}$ with a roller pump. In this study, they found no significant difference between the two groups, in terms of myocardial protection [Grover 1982]. A lot of studies are showing the importance of perfusion pressure, when giving cardioplegia. Foglia et al. reported that the heart was arrested in 19 seconds at $80 \mathrm{mmHg}$ perfusion pressure, and in 53 seconds at $30 \mathrm{mmHg}$ perfusion pressure [Foglia 1979]. In another study, Robertson et al. showed that in the case of high aortic root pressure, the cardioplegia flow from the stenotic coronary was faster and that myocardial cooling was faster and better [Robertson 1983]. Frederick et al. evaluated cardioplegia application by a roller pump and the cardioplegia application by a pressurized bag in terms of aortic root pressure. They found that the perfusion pressure that could reach the aortic root was only $31 \mathrm{mmHg}$, although the cardioplegia bag was inflated to a pressure of $300 \mathrm{mmHg}$ [Frederick 2015].

In our study, we aimed to evaluate the safety of cardioplegia application with a hand-squeezed bag, in terms of myocardial protection. For this, we evaluated the cardiac markers, and the amount of EF change at the fourth hour after the crossclamp was removed. To make a clear assessment, we chose patients with normal preoperative cardiac markers, who had elective two or three-vessel isolated coronary artery bypass surgery and had EF values above $50 \%$. When we evaluated both cardiac markers and EF changes, we found no statistical difference between both groups $(\mathrm{P}>0.05)$.

Subendocardial necrosis, which develops after cardiac surgery due to inappropriate myocardial protection, is the most important cause of morbidity and mortality. Therefore, correct cardioplegia application is very important. There are many studies in the literature evaluating cardioplegia in terms of its type, content, and temperature [Hayashida 1994]. Furthermore, many studies are comparing antegrade and retrograde cardioplegia application [Shahian 1985]. However, there are very few studies comparing cardioplegia given at certain pressure from a roller pump with cardioplegia given at an uncertain pressure with a hand-squeezed bag.

\section{CONCLUSION}

As a result of our study, cardioplegia administration with a certain constant pressure from the roller pump and handsqueezed bag with uncertain pressure does not make a difference, in terms of myocardial protection. We think that the content and amount of cardioplegia and the preferred time for repeated cardioplegia applications are more important for the protection of the myocardium. 


\section{REFERENCES}

Abah U, Garfjeld Roberts P, Ishaq M, D Silva R. 2012. Is cold or warm blood cardioplegia superior for myocardial protection? Interact Cardiovasc Thorac Surg. 14(6): 848-55.

Beyesdorf F MD, Allen BS, Bukcberg GD. 1999. Myocardial protection with integrated blood cardioplegia. In: Franco KL, Verrior ED. Editors. Advanced therapy in cardiac surgery, Hamilton: B C Decker. pp: 38-50.

Buckberg GD. 1987. Strategies and logic of cardioplegic delivery to prevent, avoid, and reverse ischemic and reperfusion damage, J Thorac Cardiovasc Surg. 93(1):127-39.

Buckberg GD, Athanasuleas CL. 2016. Cardioplegia: solutions or strategies? Eur J Cardiothorac Surg. 50(5):787-91.

Calafiore AM, Teodori G, Mezzetti A, Bosco G, Verna AM, Di Giammarco G, et al. 1995. Intermittent antegrade warm blood cardioplegia, Ann Thorac Surg. 59(2):398-402.

Candilio L, et al. 2014. A retrospective analysis of myocardial preservation techniques during coronary artery bypass graft surgery: are we protecting the heart? J Cardiothorac Surg. 9:184.

Chambers DJ. 2003. Mechanisms and alternative methods of achieving cardiac arrest. Ann Thorac Surg. 75(2):661-6.

Fan Y, Zhang AM, Xiao YB, Weng YG, Hetzer R. 2010. Warm versus cold cardioplegia for heart surgery. a meta-analysis. Eur J Cardiothorac Surg. 37(4): 912-9.

Foglia RP, Steed DL, Follette DM, DeLand E, Buckberg GD. 1979. Iatrogenic myocardial edema with potassium cardioplegia. J Thorac Cardiovasc Surg. 78(2):217-2.
Frederick L, Grover, FL, Fewel JG, Ghidoni JJ, Bennett EV, Trinkle JK. 2015. Comparison of Roller Pump versus Pressurized Bag Administration of Potassium Cardioplegic Solution. J Extra Corpor Technol. 47(4):209-16.

Grover FL, Fewel JG, Ghidoni JJ, Bennett EV,Trinkle JK. 1982. Roller pump versus pressurized bag for cardioplegia. Ann Thorac Surg. 34(3):278-86.

Hayashida N, Ikonomidis JS, Weisel RD, Shirai T, Ivanov J, Carson SM, et al. 1994. The optimal cardioplegic temperature. Ann Thorac Surg. 58(4):961-71.

Kirali K. 2006. Which application, complex or mini cardioplegia?/ Cardioplegia by minicardioplegia technique, Anadolu Kardiyol Derg. 6(3):289-91

Melrose DG, Dreyer B, Bentall HH, Baker MA. 1955. Elective cardiac arrest, Lancet. 266(2):21-3.

Robertson JM Buckberg GD, Vinten-Johansen J John M. Leaf CD. 1983. Cardioplegic delivery beyond coronary stenosis: the superiority of blood cardioplegia over asanguineous cardioplegia. J Thorac Cardiovasc Surg. 86(1): 80-6.

Shahian DM. 1985. Concepts and Techniques of Myocardial Protection for Adult Open Heart Surgery. Surg Clin North Am. 65(2):323-46.

Westaby S. 1997. Foundations of Cardiac Surgery. In: Westaby S ed.; Landmarks in Cardiac Surgery. Oxford: Isis Medical Media. 684.

Yilik L, Ozsoyler I, Yakut N, Emrecan B, Yasa H, Calli AO, et al. 2004. Passive Infusion A Simple Delivery Method for Retrograde Cardioplegia. Tex Heart Inst J. 31(4):392-7. 